\title{
Introducción a la Óptica Geométrica basada en el diseño y aplicación de un Ciclo de Mejora en el Aula
}

\section{Introduction to Geometrical Optics based on the design and the implementation of an Improvement Cycle in Classroom}

VICTORIA ESTESO CARRIZO

https://orcid.org/0000-0002-1976-8306

Universidad de Sevilla

Dpto. Física de la Materia Condensada

vesteso@us.es

DOI: http://dx.doi.org/10.12795/9788447231003.019

Pp.: 402-422 


\section{Breve descripción del contexto}

Este trabajo aborda la introducción a los fundamentos de la óptica geométrica con el diseño y aplicación de un Ciclo de Mejora en el Aula (CIMA) en la asignatura de Óptica a alumnos de Grado de Física y Doble Grado de Física y Matemáticas de la Universidad de Sevilla, en total unos 130 alumnos.

El CIMA se desarrollará durante 3 sesiones: una primera sesión de introducción, de 45 minutos, previa a dos sesiones de 3 horas cada una de ellas. Las sesiones en las que se aplica el CIMA este año, normalmente, se planifican para que tengan lugar justo antes del comienzo del laboratorio de óptica. Para los alumnos es el primer contacto con un laboratorio de óptica y las sesiones pretenden cubrir las nociones básicas necesarias para que se desenvuelvan de forma más autónoma en el laboratorio durante el desarrollo de las prácticas. Este año, por las circunstancias especiales del curso debidas a la pandemia, estas sesiones se impartirán en formato online a través de la plataforma BlackBoard Collaborate Ultra, siendo todas las sesiones sincronas.

El objetivo del CIMA consiste en dar respuesta a una pregunta problema mediante la construcción del conocimiento a través de actividades que involucran activamente a los alumnos y que se desarrollan durante las sesiones. Veremos cómo evaluación de los conocimientos previos y posteriores de los alumnos demuestran una mejora significativa tras las sesiones, y cómo el análisis de la aplicación del CIMA tiene también un impacto positivo en la mejora de la actividad docente de la profesora para el futuro.

\section{Diseño del CIMA}

El diseño de este CIMA se ha realizado siguiendo las indicaciones y actividades del Curso General de Docencia Universitaria, centrándose principalmente en trabajar en los contenidos, la metodología y la evaluación (Porlán, 2017).

Ciclos de Mejora en el Aula (2020). Experiencias de Innovación Docente de la US Esta obra se distribuye con la licencia Creative Commons 


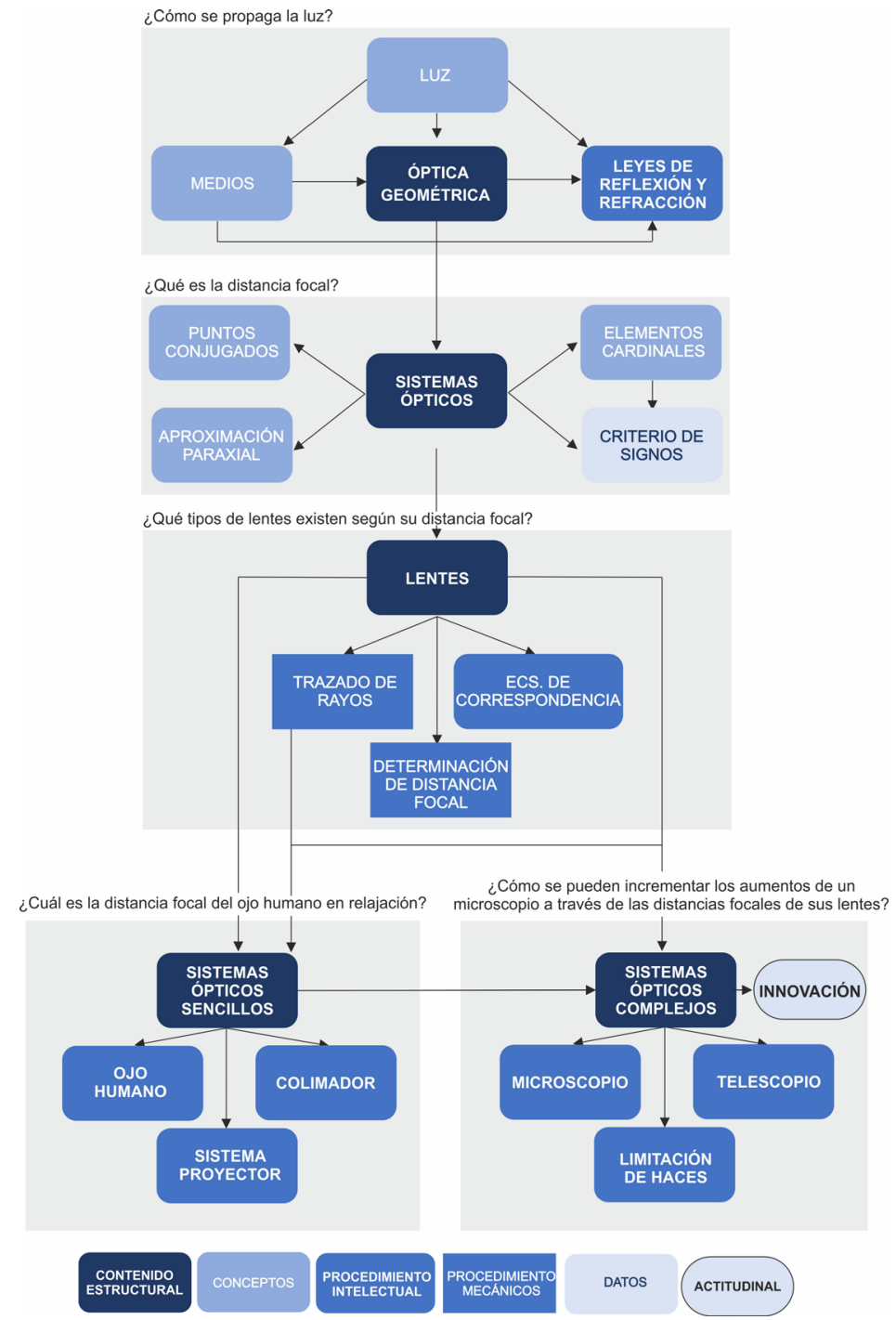

Figura 1. Mapa de contenidos del CIMA en el que se basa el diseño de la secuencia de actividades.

En primer lugar, se plantea una pregunta problema cuya respuesta abarca todos los contenidos que se quieren revisar en estas sesiones. La pregunta es: ¿Cómo se construye un microscopio con dos lupas?

Ciclos de Mejora en el Aula (2020). Experiencias de Innovación Docente de la US (c) (i) $\Theta$ Esta obra se distribuye con la licencia Creative Commons Reconocimiento-NoComercial-SinObraDerivada Internacional (CC BY-NC-ND 4.0.) 
Esta será la cuestión de fondo que motivará el proceso de construcción de conocimiento que seguirán los alumnos a lo largo de las sesiones, para finalmente darle respuesta. Para ello, se ha elaborado un mapa de contenidos (ver Figura 1) que se ha dividido en cinco secciones. Cada una de ellas se activa con una pregunta y aborda una parte específica del contenido de las sesiones. Así, poco a poco y siguiendo la secuencia de preguntas propuesta por el mapa de contenidos, se irá construyendo el conocimiento necesario para dar respuesta a la pregunta problema de este CIMA.

Es interesante observar que el contenido del mapa que se ha seleccionado (Frega, 2015), se ha clasificado según su tipología datos, conceptos, procedimientos intelectuales, procedimientos mecánicos y conocimientos actitudinales. En la parte inferior de la Figura 1 se encuentra la leyenda que hace referencia a esta clasificación. De esta forma es fácil hacerse una idea rápida de cuál es el contenido estructural, la variedad de los tipos de contenido y la fracción de contenidos de cada tipo que se revisan en estas sesiones. En particular, en este caso, el contenido inicial trata mayormente contenidos conceptuales, mientras que conforme se avanza en el mapa de contenidos, estos cambian a procedimientos intelectuales predominantemente.

\section{Modelo metodológico posible para este CIMA}

El modelo metodológico para este CIMA se dividirá en 3 fases. Una primera de introducción para presentar el problema a resolver y conocer las ideas previas de los alumnos mediante una encuesta inicial. La segunda fase corresponde con la fase conceptual y de aplicación de los contenidos teóricos. En ella, se realizarán actividades de contraste tanto prácticas como teóricas en las que los alumnos, a través de talleres y problemas simples irán familiarizándose con los conceptos y poniendo en práctica los fundamentos teóricos. Mediante la exposición de sus respuestas y la discusión de estas con el resto de 
compañeros y la profesora, se irán asentando los conocimientos e introduciendo de forma constructiva los siguientes elementos teóricos a trabajar en pequeñas dosis de teoría. Finalmente, a modo de conclusión se hará un resumen que le dé al estudiante una visión global de todo el proceso que se ha seguido.
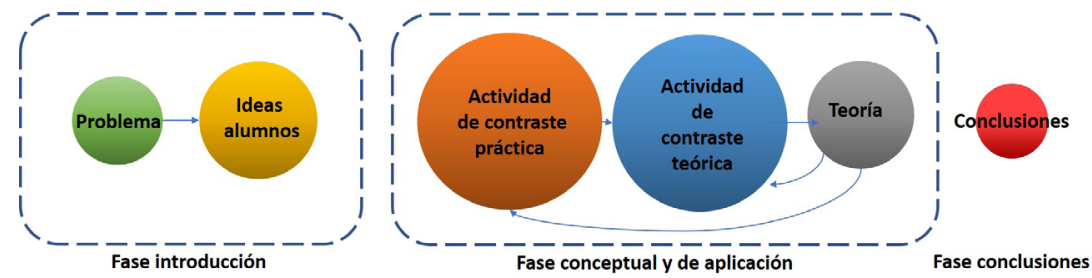

Figura 2. Esquema del modelo metodológico posible.

Secuencia de actividades.

La secuencia de actividades diseñada para el desarrollo de este CIMA se clasificará en distintos tipos de actividades:

Pregunta: Se plantea a los alumnos una pregunta inicial para que propongan sus ideas y si no saben cómo responderla que compartan cuáles creen que son los conceptos que necesitan aprender para poder responderla.

Explicación inicial: Se describe a los estudiantes en qué va a consistir la clase y cómo va a ser el desarrollo.

Encuesta inicial: Se propone a los estudiantes una serie de preguntas que les haga recapacitar sobre los conocimientos de los que parten antes de comenzar a abordar el contenido de las sesiones. Sus respuestas son recogidas y analizadas.

Multimedia: Varias de las actividades se han catalogado como multimedia ya que consiste en el visionado de vídeos o el uso de simuladores web para que los alumnos puedan 'jugar' con ellos y experimentar tratando de resolver los ejercicios propuestos en clase.

Explicación: Son píldoras breves sobre los conceptos básicos que deben manejar para poder seguir avanzado en el desarrollo de las actividades y ejercicios contenidos en los talleres por grupo.

Ciclos de Mejora en el Aula (2020). Experiencias de Innovación Docente de la US Esta obra se distribuye con la licencia Creative Commons 
Reflexión y discusión: Individualmente, los alumnos reflexionarán sobre la respuesta inicial que dieron y sobre la explicación que acaban de recibir para decidir si cambiarían o cómo modificarían su respuesta inicial. Después se pide a algunos de los alumnos que compartan con la clase cómo han cambiado sus respuestas y se abre una breve discusión entre todos por si surgen dudas que se puedan explicar unos alumnos a otros.

Talleres individuales: Los alumnos trabajan de forma individual sobre los ejercicios propuestos por la profesora para poder aportar sus propias ideas en el taller por grupos. Esto les supondrá un reto porque tendrán que aplicar los conceptos vistos en las explicaciones, relacionarlos entre sí, y poner a prueba sus razonamientos.

Talleres por grupos: En estos talleres se dividen a los estudiantes en grupos pequeños de 405 personas para tratar de resolver los ejercicios propuestos por la profesora. Tendrán que analizar cómo sus propias ideas encajan con las de los compañeros o si deben reformularse la forma en que han aprendido algunos conceptos o procedimientos intelectuales (Finkel).

Puesta en común de ideas y discusión: Cada grupo expondrá hasta donde ha llegado en la resolución de los ejercicios y qué dificultades han encontrado. La profesora animará a los alumnos que han resuelto mejor los ejercicios a que expliquen cuál ha sido la secuencia de razonamientos y si entran en conflicto con los de otros grupos.

Tabla 1. Secuencia de actividades del CIMA.

\begin{tabular}{|c|c|}
\hline Actividades (min) & Contenidos \\
\hline \multicolumn{2}{|c|}{ Sesión previa } \\
\hline \multicolumn{2}{|c|}{ Fase introducción } \\
\hline Pregunta (5) & $\begin{array}{c}\text { ¿Cómo se construye un microscopio } \\
\text { con dos lupas? }\end{array}$ \\
\hline
\end{tabular}

Ciclos de Mejora en el Aula (2020). Experiencias de Innovación Docente de la US Esta obra se distribuye con la licencia Creative Commons 


\begin{tabular}{|c|c|c|}
\hline \multicolumn{2}{|c|}{ Explicación inicial (10) } & $\begin{array}{l}\text { Descripción del contenido a tratar } \\
\text { en las sesiones y actividades a } \\
\text { realizar. }\end{array}$ \\
\hline \multicolumn{2}{|l|}{ Encuesta inicial (15) } & Encuesta inicial \\
\hline \multicolumn{2}{|l|}{ Multimedia (15) } & $\begin{array}{l}\text { Vídeo motivación (FoldScope). } \\
\text { Análisis en grupo respondiendo a } \\
\text { preguntas planteadas por profesora } \\
\text { sobre vídeo. }\end{array}$ \\
\hline \multicolumn{3}{|c|}{ Sesión 1} \\
\hline \multicolumn{3}{|c|}{ Fase conceptual } \\
\hline \multirow{4}{*}{$\begin{array}{l}\text { Actividad de } \\
\text { contraste teórica }\end{array}$} & Explicación (20) & $\begin{array}{l}\text { Óptica geométrica: ¿Cómo se } \\
\text { propaga la luz? }\end{array}$ \\
\hline & $\begin{array}{l}\text { Reflexión y } \\
\text { discusión (15) }\end{array}$ & $\begin{array}{l}\text { Discusión sobre las respuestas de la } \\
\text { primera y la segunda pregunta de la } \\
\text { encuesta inicial }\end{array}$ \\
\hline & Explicación (20) & $\begin{array}{l}\text { Sistema óptico: ¿Qué es la distancia } \\
\text { focal? }\end{array}$ \\
\hline & $\begin{array}{l}\text { Reflexión y } \\
\text { discusión (5) }\end{array}$ & $\begin{array}{l}\text { Discusión sobre las respuestas de } \\
\text { la tercera pregunta de la encuesta } \\
\text { inicial }\end{array}$ \\
\hline Teoría & Explicación (15) & $\begin{array}{l}\text { Lentes: ¿Qué tipo de lentes existen } \\
\text { según su focal? }\end{array}$ \\
\hline \multirow{4}{*}{$\begin{array}{l}\text { Actividad de } \\
\text { contraste práctica }\end{array}$} & Taller individual (10) & $\begin{array}{l}\text { Trazado de rayos en lentes delgadas. } \\
\text { Los alumnos resolverán ejercicios } \\
\text { de trazado de rayos sencillos. }\end{array}$ \\
\hline & Taller por grupos (5) & $\begin{array}{l}\text { Trazado de rayos en lentes delgadas. } \\
\text { Los alumnos compartirán los } \\
\text { resultados que han obtenido, } \\
\text { primero en grupos pequeños y } \\
\text { después en el conjunto de la clase. }\end{array}$ \\
\hline & $\begin{array}{l}\text { Comprobación } \\
\text { resultados (5) }\end{array}$ & $\begin{array}{l}\text { Link de simulador web de trazado } \\
\text { de rayos en lentes delgadas. Los } \\
\text { alumnos utilizarán el link del } \\
\text { simulador para comprobar los } \\
\text { resultados de la actividad anterior. }\end{array}$ \\
\hline & $\begin{array}{l}\text { Puesta en común y } \\
\text { discusión (10) }\end{array}$ & $\begin{array}{l}\text { Compartir con toda la clase } \\
\text { algunos ejemplos de los resultados } \\
\text { obtenidos con el simulador. }\end{array}$ \\
\hline
\end{tabular}

Ciclos de Mejora en el Aula (2020). Experiencias de Innovación Docente de la US Esta obra se distribuye con la licencia Creative Commons Reconocimiento-NoComercial-SinObraDerivada $\quad 4.0$ Internacional (CC BY-NC-ND 4.0.) 
Tabla 2. Continuación de secuencia de actividades del CIMA.

\begin{tabular}{|c|c|c|}
\hline \multicolumn{3}{|c|}{ Fase aplicaciones } \\
\hline \multirow{3}{*}{$\begin{array}{l}\text { Actividad de } \\
\text { contraste práctica }\end{array}$} & $\begin{array}{l}\text { Taller individual } \\
\text { (10) }\end{array}$ & $\begin{array}{l}\text { Diseño colimador, sistema } \\
\text { proyector, ojo humano. ¿Cuál es } \\
\text { la distancia focal del sistema } \\
\text { lente-cornea cuando está } \\
\text { relajado? }\end{array}$ \\
\hline & $\begin{array}{l}\text { Taller por grupos } \\
\text { (20) }\end{array}$ & $\begin{array}{l}\text { Compartir en pequeños grupos } \\
\text { las ideas de la actividad } \\
\text { individual y más tarde en el aula } \\
\text { en general. }\end{array}$ \\
\hline & Explicación (15) & $\begin{array}{l}\text { Fundamento colimador, sistema } \\
\text { proyector, ojo humano. }\end{array}$ \\
\hline Teoría & Multimedia (15) & $\begin{array}{l}\text { ¿Cómo medir distancia focal } \\
\text { de una lente desconocida } \\
\text { en el laboratorio? Video de } \\
\text { procedimiento en laboratorio. } \\
\text { Análisis en grupo del contenido. }\end{array}$ \\
\hline $\begin{array}{l}\text { Actividad de } \\
\text { contraste teórica }\end{array}$ & $\begin{array}{l}\text { Reflexión y } \\
\text { discusión (10) }\end{array}$ & $\begin{array}{l}\text { Discusión sobre las respuestas a } \\
\text { la cuarta pregunta de la encuesta } \\
\text { inicial. }\end{array}$ \\
\hline \multicolumn{3}{|c|}{ Sesión 2} \\
\hline Teoría & Explicación (20) & Conjuntos de lentes. Conceptos. \\
\hline \multirow{3}{*}{$\begin{array}{l}\text { Actividad de } \\
\text { contraste práctica }\end{array}$} & $\begin{array}{l}\text { Taller individual } \\
\text { (15) }\end{array}$ & $\begin{array}{l}\text { Trazado de rayos de conjunto } \\
\text { de lentes. Los estudiantes } \\
\text { resolverán ejercicios de trazados } \\
\text { de rayos más complejos. }\end{array}$ \\
\hline & $\begin{array}{l}\text { Taller en grupo } \\
\text { (15) }\end{array}$ & $\begin{array}{l}\text { Puesta en común en grupos de } \\
\text { los resultados obtenidos. }\end{array}$ \\
\hline & Multimedia (10) & $\begin{array}{l}\text { Simulador de rayos en } \\
\text { extensión Chrome. Los alumnos } \\
\text { experimentarán con otro } \\
\text { simulador y tratarán de resolver } \\
\text { los ejercicios de trazado de rayos } \\
\text { utilizándolo. }\end{array}$ \\
\hline $\begin{array}{l}\text { Actividad de } \\
\text { contraste teórica }\end{array}$ & $\begin{array}{l}\text { Taller por grupos } \\
\text { (20) }\end{array}$ & $\begin{array}{l}\text { ¿Cómo construir un microscopio } \\
\text { con dos lentes? }\end{array}$ \\
\hline
\end{tabular}

Ciclos de Mejora en el Aula (2020). Experiencias de Innovación Docente de la US Esta obra se distribuye con la licencia Creative Commons Reconocimiento-NoComercial-SinObraDerivada Internacional (CC BY-NC-ND 4.0.) 


\begin{tabular}{|c|l|l|}
\hline \multirow{2}{*}{ | } & $\begin{array}{c}\text { Puesta en común } \\
\text { y discusión (15) }\end{array}$ & $\begin{array}{l}\text { Puesta de ideas en común y } \\
\text { discusión sobre las respuestas a } \\
\text { la quinta pregunta de la encuesta } \\
\text { inicial }\end{array}$ \\
\cline { 2 - 3 } & Explicación (15) & Fundamentos microscopio \\
\hline Teoría & Explicación (15) & ¿Cómo funciona telescopio? \\
\hline \multirow{2}{*}{ Actividad de } & Explicación (20) & $\begin{array}{l}\text { Limitación de haces: pupilas y } \\
\text { lucarnas }\end{array}$ \\
\hline contraste práctica & $\begin{array}{l}\text { Taller por grupos } \\
\text { (15) }\end{array}$ & $\begin{array}{l}\text { Diafragmas. Pupilas y lucarnas } \\
\text { con simulador óptico. Los } \\
\text { estudiantes experimentan por } \\
\text { grupos con estos conceptos en el } \\
\text { simulador. }\end{array}$ \\
\cline { 2 - 3 } & $\begin{array}{l}\text { Puesta en común } \\
\text { y discusión (10) }\end{array}$ & $\begin{array}{l}\text { Puesta de ideas en común y } \\
\text { discusión resultados sobre el } \\
\text { ejercicio de pupilas y lucarnas. }\end{array}$ \\
\hline \multicolumn{2}{|c|}{ Fase conclusiones } \\
\hline \multirow{2}{*}{ Explicación (10) } & $\begin{array}{l}\text { Resumen y conclusiones del } \\
\text { contenido trabajado }\end{array}$ \\
\hline
\end{tabular}

Cuestionario de diagnóstico inicial-final.

Para conocer los modelos mentales de los estudiantes se planteó un cuestionario al inicio y al final del CIMA. Para ello se utilizaron las plataformas 'Socrative' y 'Google Forms'. Las preguntas de los cuestionarios fueron las siguientes.

1. ¿Cuál es para ti la diferencia entre la óptica geométrica y la óptica ondulatoria? ¿Qué puntos crees que tienen en común y cuáles son las diferencias?

2. Describe lo más detallado posible cómo es la propagación de la luz a través del cristal de una ventana y qué fenómenos se dan en ese proceso. ¿Qué es el ángulo límite?

3. Inicial: ¿Qué significa para ti focalizar la luz?

4. Final: ¿Qué es la distancia focal de una lente o un sistema? ¿Cuál es la distancia focal del ojo humano cuando está relajado y por qué?

Ciclos de Mejora en el Aula (2020). Experiencias de Innovación Docente de la US Esta obra se distribuye con la licencia Creative Commons 
5. Cuando uno tiene una lente en el laboratorio sin etiquetar, ¿cómo piensas que se puede averiguar el valor de las dioptrías de la lente?

6. a) ¿Cómo crees que se construye un microscopio óptico con lentes? b) ¿Cómo crees que se consigue aumentar tanto el tamaño de la imagen de un objeto con un microscopio?

\section{Aplicación del CIMA}

A continuación, se describe el desarrollo de las sesiones durante la aplicación del CIMA.

\section{Sesión previa (45 min):}

En esta sesión previa comienzo explicando cómo se desarrollarán las siguientes sesiones y dado que será algo diferente a lo que puedan estar acostumbrados les pido su compromiso para participar de forma activa en las actividades que se propongan.

A continuación, decido comenzar con la encuesta inicial, a través de Google Forms y Socrative, para la que los alumnos dispondrán de 15 minutos para resolverla. Una vez han rellenado la encuesta, les explico que las sesiones van a girar en torno a un problema y que todo el contenido de las sesiones va dirigido a construir el conocimiento necesario para dar respuesta a esa pregunta.

En ese momento, les propongo ver un vídeo introductorio para motivar el problema. Se trata de un video de YouTube de una charla TED de 2012 sobre la invención de un microscopio que se construye en papel como un origami (Foldscope). Les pido que respondan a tres preguntas: 1) ¿Cuál es el problema que se resuelve?, 2) ¿Cuál es el componente innovador de la propuesta?, y 3) ¿Qué elemento no cambian y por qué? A pesar del temor a que no hayan encontrado interesante la actividad, los alumnos sí que se muestran participativos y motivados a la hora de responder las preguntas.

Ciclos de Mejora en el Aula (2020). Experiencias de Innovación Docente de la US Esta obra se distribuye con la licencia Creative Commons 
Seguidamente, hago un resumen de las respuestas que han dado y completo algún aspecto que no se ha mencionado para finalizar indicando que, aunque el contenido de las sesiones que se van a trabajar a continuación es conocido desde hace más de 200 años, a día de hoy la innovación aplicada a esos conocimientos pueden resolver problemas a nivel mundial como el diagnóstico de enfermedades causadas por bacterias en países con menos recursos, como han visto en el vídeo.

Para finalizar la sesión previa, termino por enseñarles el mapa de contenidos para que tengan un esquema del camino que seguiremos en las siguientes sesiones.

\section{Sesión 1 (3 horas):}

Al comienzo de la primera sesión, la primera actividad consiste en ir resolviendo en orden las preguntas de la encuesta inicial, profundizando en la teoría que hay detrás de cada una de ellas. Así comienzo explicando las diferencias entre óptica geométrica y ondulatoria y cómo se propaga la luz cuando atraviesa medios materiales. Durante la explicación, por tratarse de una sesión online, no tengo ninguna retroalimentación por parte de los alumnos, no puedo verlos y no sé cuáles están siendo sus reacciones y aunque cuando pregunto responden que todo va bien, echo de menos tener la información que se tiene cuando la clase es presencial.

Tras las explicaciones les pido que reflexionen sobre cómo respondieron a las preguntas relacionadas en el cuestionario inicial y cómo responderían ahora después de escuchar la explicación teórica. Temo de nuevo que la participación sea baja y efectivamente, sólo algún alumno responde y de forma simple. No sé muy bien a qué se debe esa baja participación. Prefiero no darle importancia y continuar con la siguiente actividad sobre trazado de rayos que es más entretenida.

Explico la teoría necesaria para comenzar con la actividad de trazado de rayos, proponiendo los ejercicios que 
ellos deben resolver. Primero deben trabajar de forma individual tratando de resolver los ejercicios por ellos mismos y a continuación en la plataforma BlackBoard, distribuyo a los alumnos en pequeños grupos de 405 alumnos para que pongan en común los resultados a los que han llegado o para que planteen a sus compañeros las dudas que les impiden avanzar.

Mientras tanto, la plataforma me permite unirme a cada uno de los grupos y voy visitando algunos de ellos para formar parte de la discusión de forma pasiva, interviniendo sólo cuando parece que la discusión no avanza (Finkel, 2008).

Después hacemos una puesta en común de la resolución de los ejercicios en la sala principal de la plataforma. Para ello trato de crear un entorno en el que se sientas seguros exponiendo sus respuestas, pero que a la vez fomente el aprendizaje crítico (Bain, 2005). Comienzo preguntando a los grupos que han tenido más dificultades y que, o bien se han quedado bloqueados y no han podido terminar algunos de los ejercicios. Los alumnos comparten su pantalla con el resto de la clase y cuentan cuál es el razonamiento que han seguido. Me sorprende que son los propios alumnos los que cuentan qué errores estaban cometiendo para prevenir a otros compañeros. Los animo a que se corrijan entre sí e intervengo sólo cuando es necesario.

Quedo muy satisfecha del desarrollo de esta actividad pues creo que ha superado mis expectativas. Sin embargo, me veo obligada a interrumpir una actividad para no perder el tiempo a pesar de que creo que está siendo muy productiva. Lamento no haber programado más tiempo para ella.

Por último, antes de acabar la sesión muestro unos vídeos que he grabado en el laboratorio donde procedo a realizar una parte de las prácticas que deben llevar a cabo ellos más adelante, para explicarles los diferentes métodos que tiene que aprender a realizar en el laboratorio. Por falta de tiempo, la discusión sobre este contenido me veo obligada a suprimirla.

Ciclos de Mejora en el Aula (2020). Experiencias de Innovación Docente de la US Esta obra se distribuye con la licencia Creative Commons 
Sesión 2 (3 horas):

Para comenzar la segunda sesión, vuelvo a mostrar el mapa de contenidos incidiendo en los conceptos más importantes que se necesitan recordar para continuar con la sesión.

En primer lugar, explico la teoría relacionada con conjuntos de lentes relacionándolo con lo que vimos en la sesión anterior para lentes delgadas y vemos algunos ejemplos de trazado de rayos en esos sistemas más complejos. Cuando termino la explicación, espero que lo alumnos hagan preguntas para resolver sus dudas, pero apenas intervienen.

Presento el ejercicio en el que van a trabajar y que consiste en dibujar el trazado de rayos de un doblete de lentes y averiguar dónde se forma la imagen final que crea el sistema óptico. Les explico que existen tres métodos para resolverlo: dibujando el trazado de rayos, utilizando las ecuaciones presentadas o utilizando un simulador de trazado de rayos (https://ricktu288.github.io/ray-optics/). Les explico brevemente cómo se utiliza el simulador y les pido que, en primer lugar, traten de resolver el problema individualmente utilizando el método que más sencillo les resulte y que después, trabajen en los pequeños grupos. Pido que en cada grupo de trabajo se resuelva el problema utilizando los tres métodos y que comparen los resultados obtenidos empleando los diferentes métodos.

De nuevo, voy visitando los grupos de trabajo e interviniendo cuando los alumnos lo piden. Este ejercicio parece costarles más, pero muestran interés por resolverlo ellos mismos. Por este motivo, les concedo más tiempo del establecido porque creo que lo están aprovechando. Transcurrido el tiempo, disuelvo los grupos de trabajo y nos encontramos todos en la sala principal. En ese momento muchos de los alumnos comentan en el chat que no han sabido llegar a la solución utilizando las expresiones matemáticas y que tienen interés en ver dónde se han equivocado. Pido a los grupos que no han conseguido

Ciclos de Mejora en el Aula (2020). Experiencias de Innovación Docente de la US Esta obra se distribuye con la licencia Creative Commons 
resolver el problema que planteen los bloqueos que han encontrado y cuando lo hacen pido a los grupos que sí lo han resuelto que ayuden a sus compañeros. Aunque generalmente las explicaciones que dan los alumnos suelen ser correctas, a veces intervengo para terminar de completarlas o para recalcar una idea. Comparto mi pantalla con la solución utilizando las ecuaciones y explico paso a paso el procedimiento. Algunos de los alumnos indican dónde se habían equivocado ellos. Siento que la actividad ha sido bastante fructífera y que los alumnos han quedado satisfechos con el ejercicio. Es entonces cuando les sorprendo diciendo que ya han construido su primer microscopio, que el ejercicio que hemos resuelto es el ejemplo más sencillo de microscopio y entonces analizamos las características del instrumento y lo relaciono con las explicaciones anteriores.

Paso en ese momento a explicar rápidamente los fundamentos del microscopio y del telescopio, para llegar antes de acabar la sesión a explicar al menos la parte teórica sobre limitación de haces. No me da tiempo a plantear la actividad para esa última parte y prefiero terminar haciendo un breve repaso de lo visto en las sesiones para terminar. Me queda un sabor agridulce por no haber podido ir con más calma en la última parte, pero a la vez estoy contenta porque he visto cómo han trabajado los alumnos en las actividades anteriores y cómo han ido asentando y aplicando conceptos en las actividades realizadas.

Este CIMA ha sido observado por otro profesor durante su aplicación. En su informe, destaca como puntos positivos la presentación del mapa de contenidos y la definición clara de los contenidos que son necesarios aprender, y la supervisión de la profesora durante la puesta en común, donde son los propios alumnos los que instruyen a los compañeros explicando sus procedimientos. Como puntos a mejorar propone explicar con mayor claridad el problema que se va a resolver durante las sesiones y no dar el contenido conceptual directamente sino construirlo a partir del conocimiento de los alumnos.

Ciclos de Mejora en el Aula (2020). Experiencias de Innovación Docente de la US Esta obra se distribuye con la licencia Creative Commons 


\section{Evaluación del aprendizaje de los estudiantes}

En esta sección se presenta un ejemplo del análisis de las preguntas de la encuesta inicial y final y se muestra una tabla que recoge la evolución en el aprendizaje de los alumnos.

Pregunta 1: ¿Cuál es para ti la diferencia entre óptica geométrica y óptica ondulatoria? ¿Qué puntos tiene en común y cuáles crees que son las diferencias?

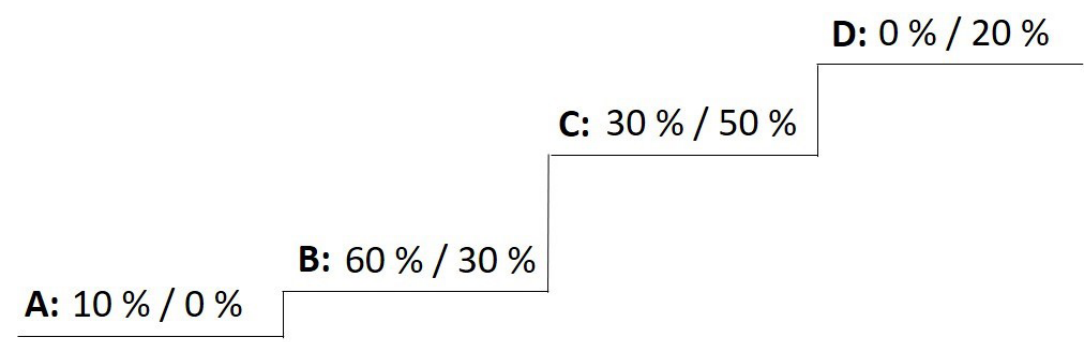

Figura 3. Escalera de aprendizaje de la pregunta 1.

Tabla 3. Clave de la escalera de aprendizaje de la pregunta 1.

\begin{tabular}{c|l|l|}
\hline Nivel & Conocimiento & Obstáculo \\
\hline A & Ignora naturaleza dual de luz & $\begin{array}{l}\text { Descripción de luz como onda y } \\
\text { como corpúsculo }\end{array}$ \\
\hline B & $\begin{array}{l}\text { Identifica luz como rayo u onda } \\
\text { para cada teoría. }\end{array}$ & $\begin{array}{l}\text { Características de la } \\
\text { descripción geométrica u } \\
\text { ondulatoria de la luz. Cada } \\
\text { teoría permite explicar } \\
\text { comportamientos diferentes de } \\
\text { la luz. }\end{array}$ \\
\hline C & $\begin{array}{l}\text { Comprende que cada teoría } \\
\text { puede describir fenómenos } \\
\text { diferentes. }\end{array}$ & $\begin{array}{l}\text { Existencia de fenómenos como } \\
\text { la interferencia, difracción, } \\
\text { posibles sólo por la naturaleza } \\
\text { ondulatoria de la luz. }\end{array}$ \\
\hline D & $\begin{array}{l}\text { Nombra fenómenos que se } \\
\text { pueden describir con cada } \\
\text { teoría y por qué. }\end{array}$ & \\
\hline
\end{tabular}

Ciclos de Mejora en el Aula (2020). Experiencias de Innovación Docente de la US Esta obra se distribuye con la licencia Creative Commons 
Las respuestas de los alumnos a las preguntas de las encuestas han sido clasificadas en una serie de modelos mentales A, B, C, D que gradualmente incremental el nivel de complejidad, desde el más sencillo (A) al más completo (D). Los valores porcentuales indican el porcentaje de alumnos cuya respuesta está incluida en cada nivel de complejidad del modelo mental. El valor de la izquierda corresponde a la respuesta en la encuesta inicial y el de la derecha, a la respuesta de la encuesta final. La tabla bajo la escalera de aprendizaje, describe el contenido asociado a cada modelo mental (columna de en medio) y el obstáculo asociado para pasar al siguiente nivel de modelo mental (columna de la derecha) (Rivero y Porlán, 2017).

De forma generalizada, se observa que en todas las escaleras el porcentaje de la encuesta final aumenta en los niveles más altos y disminuye en los niveles más bajos de modelos mentales. Esto indica una evolución en el aprendizaje del alumnado en general. Sin embargo, el porcentaje de la encuesta final en los niveles más bajos de modelos mentales no siempre resulta ser cero, que sería el objetivo ideal al que se aspira.

La tabla 4 representa, en escala de colores donde el amarillo es el nivel más bajo y el rojo el más alto, los modelos mentales asociados a las respuestas a la encuesta inicial y final de un conjunto de alumnos.

Tabla 4. Análisis individual de la evolución en las escaleras de aprendizaje.

\section{Preguntas \\ Encuesta inicial \\ Encuesta final}

\begin{tabular}{l|l|l|l|l|l|l|l|l|l|l|l|l|l|l|l|l|l|l|l}
1 & 2 & 3 & 4 & 5 & 6 & 7 & 8 & 9 & 10 & 1 & 2 & 3 & 4 & 5 & 6 & 7 & 8 & 9 & 10
\end{tabular}

Ciclos de Mejora en el Aula (2020). Experiencias de Innovación Docente de la US Esta obra se distribuye con la licencia Creative Commons 


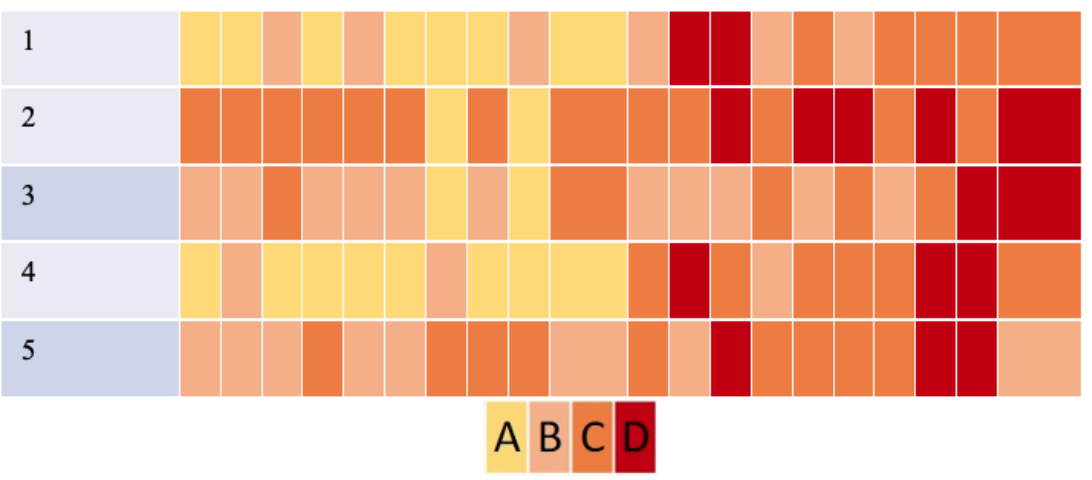

Como se anticipaba, predominan los modelos mentales más complejos en las respuestas a la encuesta final, comparados con las respuestas a la encuesta inicial. Siguiendo el orden de izquierda a derecha los alumnos han superado el siguiente número de escalones en las escaleras: $3,5,6,2,5,5,5,7,7,5$. En este recuento no se ha tenido en cuenta la pregunta 3, de modo que el siguiente análisis se ha realizado para 4 preguntas en total. Los alumnos que han superado menos de 4 escalones (20\% de los alumnos), serán los que menos han evolucionado ya que no han conseguido avanzar sus modelos mentales al menos un nivel cada uno. Los alumnos que ha superado 4 escalones o más (80\% de los alumnos), han conseguido evolucionar sus modelos mentales, en promedio, al menos un escalón en cada pregunta. Sin embargo, no hay alumnos que hayan superado 8 escalones o más, para los que se podría decir que serían los que mejor habrían aprovechado la clase, ya que, en promedio, habrían evolucionado sus modelos mentales en dos niveles en cada pregunta.

La conclusión con este análisis es que en general existe una evolución en los modelos mentales de los alumnos tras las sesiones en las que se aplica el CIMA y de forma individual se demuestra que un gran porcentaje de los alumnos evoluciona en promedio al menos un escalón en cada pregunta. Sin embargo, no se observan alumnos que hayan evolucionado en promedio dos escalones por pregunta en sus modelos mentales, lo que indicaría un muy

Ciclos de Mejora en el Aula (2020). Experiencias de Innovación Docente de la US Esta obra se distribuye con la licencia Creative Commons 
buen aprovechamiento de las sesiones. En vista de estos resultados, se debe trabajar con el objetivo de aumentar el número de alumnos que superan 2 escalones en promedio por pregunta y reducir los alumnos que no llegan en promedio a superar 1 escalón por pregunta.

\section{Evaluación del CIMA}

Tras la reflexión sobre la aplicación del CIMA, sin duda me quedo con el trabajo en grupo de los alumnos en la resolución de ejercicios. Creo que ha sido el tiempo mejor aprovechado. Sin embargo, suprimiría las discusiones sobre las respuestas de la encuesta inicial tras ver en clase las explicaciones teóricas. Quizás los alumnos no tienen tiempo suficiente para asentar los contenidos y necesitan una reflexión más profunda.

Junto a esto, me ha gustado mucho ver cómo en esta experiencia guiada por el Curso General de Docencia Universitaria, se ha ensalzado la importancia de las ideas y los modelos mentales de los alumnos y la necesidad de hacer una encuesta inicial siempre y cuando después se haga el análisis adecuado de los resultados. Diferenciar y jerarquizar los distintos modelos mentales de los alumnos para construir las escaleras de aprendizaje me ha hecho reflexionar mucho sobre cómo aprenden los estudiantes y qué información se les debe proporcionar para que superen los obstáculos y alcancen los objetivos de la asignatura. Por estos motivos, mi docencia a partir de ahora me gustaría diseñarla teniendo en cuenta los modelos mentales de los alumnos.

En segundo lugar, repensar los contenidos que se enseñan en la clase discriminando entre contenido estructural y complementario, me ha hecho salir de la dinámica asociada a cumplir un programa docente establecido que a menudo trata todos los contenidos con el mismo nivel

Ciclos de Mejora en el Aula (2020). Experiencias de Innovación Docente de la US Esta obra se distribuye con la licencia Creative Commons 
de importancia. Me he reencontrado con los mapas conceptuales, descubriendo que son una herramienta muy potente para ver, rápidamente, la relación entre diferentes contenidos y que pueden servir de guía inicial para los alumnos. Además, pensar preguntas problema que motivan y activan regiones del mapa de contenidos. Por tanto, me quedo para mi docencia la idea de construir los mapas de contenido que muestren las relaciones entre los diferentes contenidos y que tengan preguntas activadoras de regiones del mapa. Creo que pueden ser útiles tanto para mí misma como para compartirlo con los alumnos y esquematizar el desarrollo de las clases.

En tercer lugar, he tomado conciencia del modelo metodológico que estaba siguiendo Me ha motivado mucho descubrir la importancia de las fases de contraste durante el desarrollo de la clase, que solo son posibles si previamente existe un problema que resolver. Me planteo incluir en mi docencia clases que se articulen con la resolución de un problema y en las que aplique un modelo metodológico que incluya fases de contraste, ya que, por lo que he experimentado con este curso, los alumnos aprovechan y avanzan mucho más así que con el modelo metodológico de corte más transmisivo.

Finalmente, las escaleras de aprendizaje no sólo me han permitido ver la evolución de los modelos mentales de los estudiantes sino, de algún modo, cuantificar también el impacto de mi docencia sobre el conjunto de la clase. Me ha ayudado escribir un diario (Porlán, 2008) para juzgar qué partes han ido mejor de lo que esperaba y qué partes no se han desarrollado como había imaginado. Para mi docencia, me quedo con la idea de escribir de forma habitual un breve diario al finalizar las sesiones para evaluar de forma cualitativa mi docencia, y quizás de una forma más excepcional por el trabajo que supone, el análisis detallado de las escaleras de aprendizaje para la evaluación cuantitativa del desarrollo de las clases.

Ciclos de Mejora en el Aula (2020). Experiencias de Innovación Docente de la US Esta obra se distribuye con la licencia Creative Commons 
En su conjunto, esta experiencia ha sido muy positiva y con sus fundamentos impulsa significativamente la calidad de la actividad docente.

Palabras clave: Física, docencia universitaria, óptica geométrica, innovación docente, experimentación docente universitaria.

Keywords: Physics, University Teaching, Geometrical Optics, Teaching Innovation, University Teaching Experimentation,

Ciclos de Mejora en el Aula (2020). Experiencias de Innovación Docente de la US Esta obra se distribuye con la licencia Creative Commons 


\section{Referencias Bibliográficas}

Bain, K. (2005). Lo que hacen los mejores profesores universitarios. Valencia: Universidad de Valencia.

Finkel, D. (2008). Dar clases con la boca cerrada. Valencia: Publicaciones Universidad de Valencia

Frega, D.J. (2015). Recorte, selección y organización de contenidos. Escritos en la Facultad, 109, 13-15.

Porlán, R. (2008). El diario de clase y el análisis de la práctica. Averroes: Red telemática educativa de Andalucía.

Porlán, R. (Coord.) (2017). Enseñanza Universitaria. Cómo mejorarla. Madrid: Morata/ Ediciones. Universidad de Sevilla.

Rivero, A. y Porlán, R. (2017). La evaluación en la enseñanza universitaria. En R. Porlán Ariza (Coord.), Enseñanza Universitaria. Cómo mejorarla (pp. 73-91). Madrid: Morata/ Ediciones. Universidad de Sevilla.

Ciclos de Mejora en el Aula (2020). Experiencias de Innovación Docente de la US Esta obra se distribuye con la licencia Creative Commons 